\title{
Research on the effects of idling start-stop function on light vehicles fuel consumption and emission under different cycle conditions
}

\author{
Zhicheng Ma*, Tieqiang Fu, Yuwei Wang, Wei Zhao, and Luowei Zhang \\ China Automotive Technology and Research Center Co., Ltd., Tianjin 300300, China
}

Keywords: idling start-stop function, cycle conditions, light gasoline vehicles, emissions, fuel consumption.

\begin{abstract}
The idling distribution characteristics of NEDC, WLTC and CLTC conditions were analyzed, and the exhaust emissions and fuel consumption of three light gasoline vehicles when the idling start-stop function was turned on and off under different cycle conditions were measured. The effects of idling start-stop function on light vehicle fuel consumption and emissions under different cycle conditions were analyzed. The results show that the vehicle fuel saving rate of the idling start-stop function in three cycle conditions is WLTC, NEDC and CLTC conditions from low to high. The idling start-stop function has little effect on vehicle gaseous pollutant emissions. On the whole, the the activation of idling start-stop function increases the THC and $\mathrm{CO}$ emissions and reduces $\mathrm{NOx}$ emissions.
\end{abstract}

$\begin{array}{ll}\text { Abbreviations } & \\ \text { NEDC } & \text { New European Driving Cycle } \\ \text { GDI } & \text { Gasoline Direct-Injection } \\ \text { WLTC } & \text { Worldwide Light-duty Test Cycle } \\ \text { TWC } & \text { Three Way Catalytic Converter } \\ \text { CLTC } & \text { China Light-duty Vehicle Test Cycle } \\ \text { GPF } & \text { Gasoline Particulate Filter }\end{array}$

\section{Introduction}

In the past ten years, with the gradual increase of China's urbanization rate and the rapid growth of the national economy, China's auto industry output value and car ownership have shown rapid growth. The rapid development of the automotive industry has also brought huge energy consumption and environmental pollution [1-3].In order to alleviate this pressure, China has successively formulated and released a series of test methods and limit standards aimed at improving the fuel economy of automobiles, formulated and implemented policies for energy-saving automobile promotion subsidies, vehicle and vessel

\footnotetext{
*Corresponding author: mazhicheng@catarc.ac.cn
} 
tax reductions, etc., which effectively promoted the introduction of advanced technologies and development, idling start-stop technology is one of the key technologies. As we all know, since China's implementation of light vehicle emission test standards, it has been using the European NEDC condition until the China 6 emission standard was changed to WLTC condition. At present, the fuel consumption test is still using NEDC condition. However, factors such as complex land area and different urban planning in China determine that China has unique condition with Chinese characteristics. Neither NEDC or WLTC conditions can represent the actual situation in China. In this case, China's relevant departments have established their own light vehicle test cycle condition (CLTC) [4-5]. In November 2019, the "China working condition" development and application technology research conference was held in Nanjing, which established that all models after 2025 will adopt the CLTC condition. The current research on energy saving and emission reduction of idling start-stop technology still stays in NEDC condition [6-8], but WLTC and CLTC conditions are quite different from NEDC condition, and how idling start-stop technology plays a role in WLTC and CLTC conditions is rarely studied. This article mainly studies the performance of light vehicles idling start-stop technology in different cycle conditions, provides corresponding data and theoretical basis for automobile manufacturers, and prepares for the subsequent emission and fuel consumption switching WLTC and CLTC conditions.

\section{Test equipment and test plan}

\subsection{Test plan}

In this paper, three light gasoline vehicles with idling start-stop function and meeting the China 6 emission standard were selected as test vehicles to measure the emissions and fuel consumption of each vehicle when the idling start-stop function was turned on and off under NEDC,WLTC and CLTC conditions respectively. All tests were completed by the same driver, and the temperature and relative humidity set in the environmental chamber were 25 degree Celsius and 50\%. The resistance setting values of the chassis dynamometer corresponding to each test condition was the same. Before the test, the vehicle battery was charged to ensure that the battery power was the same during the test, and the vehicle shall be fully immersed in the environment of 25 degree Celsius. The temperature of the engine oil and the coolant must be within the range of $25 \pm 2$ degree Celsius, so as to ensure that the vehicle status was consistent at the beginning of the test. Eliminate the influence of drivers, environmental conditions, battery power, vehicle status and chassis dynamometer resistance on the test, and ensured that the single variable of the test was the idling start-stop function.

\subsection{Selection of test vehicles}

The In this paper, three test vehicles were selected, which are made in China, Germany and Sino foreign joint venture, and their engine displacements are not the same.Their specific parameters are shown in Table 1.

Table 1. Parameters of test vehicles.

\begin{tabular}{cccccc}
\hline Vehicle & $\begin{array}{c}\text { Displacements } \\
(\mathrm{L})\end{array}$ & $\begin{array}{c}\text { Curb weight } \\
(\mathrm{kg})\end{array}$ & $\begin{array}{c}\text { Intake } \\
\text { mode }\end{array}$ & Spray & $\begin{array}{c}\text { After treatment } \\
\text { system }\end{array}$ \\
\hline 1 & 1.497 & 1592 & Turbo & GDI & TWC+GPF \\
2 & 1.969 & 1738 & Turbo & GDI & TWC \\
3 & 2.894 & 1840 & Turbo & GDI & TWC+GPF \\
\hline
\end{tabular}




\subsection{Test equipment}

The relevant tests in this article were completed at China Automotive Technology and Research Center Co., Ltd., and related equipment information is shown in Table 2.

Table 2. Test equipment related information.

\begin{tabular}{ccc}
\hline Equipment & Model & Manufacturer \\
\hline Environmental chamber & WK690/40 & Germany WEISS \\
Analyzer & MEXA-7200H & Japan HORIBA \\
Chassis dynamometer & ROADSIM 48”4X4 & Austria AVL \\
Constant volume dilution & CVS-7400T & Japan HORIBA \\
\hline
\end{tabular}

\section{Cycle condition and idling distribution characteristics}

\subsection{Cycle conditions}

The cycle conditions in this paper are NEDC, WLTC and CLTC, as shown in Figure 1. The NEDC condition is composed of 4 urban cycles and 1 suburban cycle, with a maximum speed of $120 \mathrm{~km} / \mathrm{h}$, and the total cycle time is $1180 \mathrm{~s}$. The WLTC condition is composed of four stages of low speed, medium speed, high speed, and ultra high speed, and the maximum speed is $131.3 \mathrm{~km} / \mathrm{h}$, and the total cycle time is $1800 \mathrm{~s}$. CLTC condition consists of three stages of low speed, medium speed and high speed, the maximum speed is $114 \mathrm{~km} / \mathrm{h}$, and the total cycle time is $1800 \mathrm{~s}$.

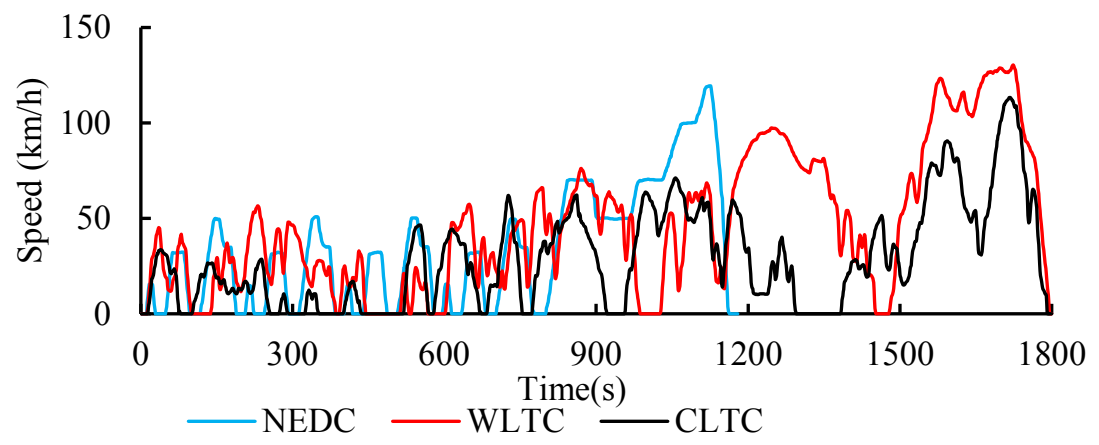

Fig. 1. Cycle conditions.

\subsection{Distribution characteristics of idling under different cycle conditions}

Table 3 shows the times of idling and the distribution of idling time for three cycles. It can be seen that the idling times of NEDC condition are the most, the idling time of CLTC condition is the longest, the idling times and the total idling time of WLTC condition are the lowest of the three cycles.

Table 3. Idling distribution characteristics.

\begin{tabular}{lccc}
\hline & NEDC & WLTC & CLTC \\
\hline Times of idling & 14 & 8 & 12 \\
Total idling time(s) & 280 & 235 & 419 \\
Proportion & $23.7 \%$ & $13.1 \%$ & $23.3 \%$ \\
\hline
\end{tabular}

The total idling time is composed of a series of idling segments. The duration of idling segments varies from several seconds to 90 seconds. The difference in the composition of 
idling segment duration will also lead to different fuel saving performance. Figure 2 shows the time of each idling segment under three cycles. Take $5 \mathrm{~s}$ as the step to count the time distribution of idling segments under three cycles, as shown in Figure 3. It can be seen that the idling distribution of NEDC condition is relatively uniform, and it is more than $10 \mathrm{~s}$ but less than 30 s, in which the idling segment with a duration of $20-25 \mathrm{~s}$ accounts for $67.14 \%$ of the total idling. In WLTC condition, there are three idling segments with short duration. The duration of the 3rd, 5th and 9th idling segments are all less than $10 \mathrm{~s}$, which are $6 \mathrm{~s}, 3 \mathrm{~s}$ and $6 \mathrm{~s}$ respectively, accounting for $6.38 \%$ of the total idling. The idling segments with more than $45 \mathrm{~s}$ account for $28.51 \%$ of the total idling. In CLTC condition, all idling segments are $10 \mathrm{~s}$ or more, in which the 6 th and 11 th idling segments are up to $85 \mathrm{~s}$ or more, and the proportion of idling segments over $45 \mathrm{~s}$ is $54.65 \%$.

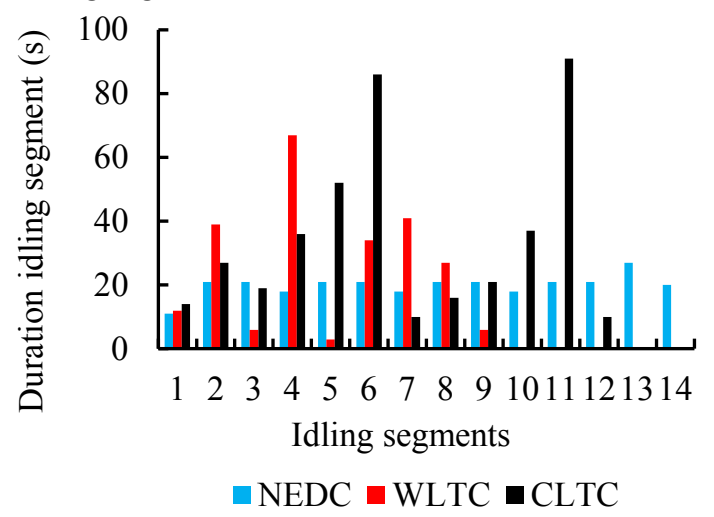

Fig. 2. Duration distribution of idling segments.

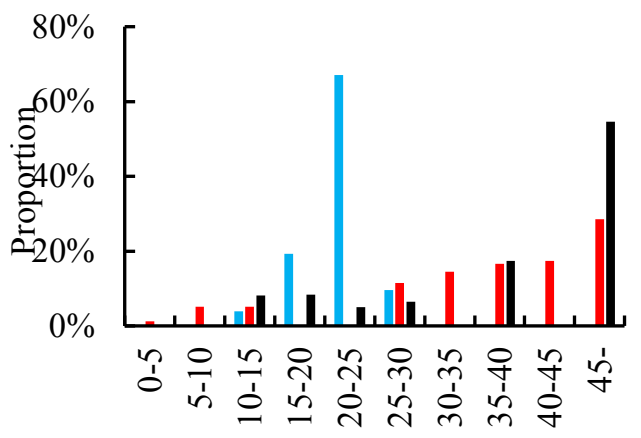

Idling segment duration (s)

$\square$ NEDC $\square$ WLTC $\square$ CLTC

Fig. 3. Proportion distribution of idling segments with different duration.

\section{Results and analysis}

\subsection{Effect of idling start-stop function on fuel consumption of different cycles}

It was found that the instantaneous fuel consumption of three vehicles in different cycles were the same when the idling start-stop function was turned on and off. Vehicle 2 was used as an example for specific explanation. Figures 4 to 6 show the instantaneous fuel 
consumption of the NEDC condition, WLTC condition and CLTC condition of the vehicle 2 respectively. It can be seen from the figure that when the idling start-stop function was turned off, all the idling start-stop functions of idling segments are not working. When the idling start-stop function was turned on, the idling start-stop function of the first idling segment of the NEDC condition did not work normally. This was because the coolant temperature was too low at this time, which did not reach the threshold to trigger the idling start-stop operation. In addition, the idling start-stop function of the rest idling segments of the three cycles worked normally. In addition to the idling segment, the instantaneous fuel consumption of the vehicle was basically the same when the idling start-stop function was turned on and off.

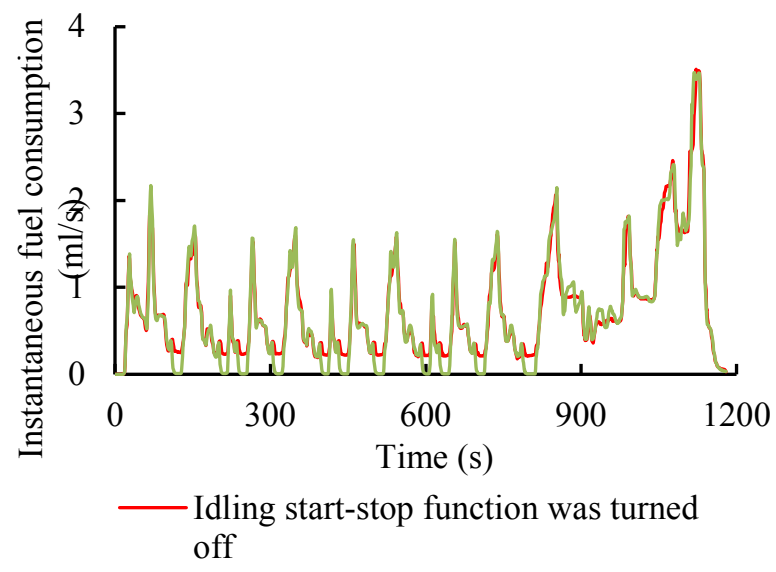

Fig. 4. Instantaneous fuel consumption of NEDC condition test.

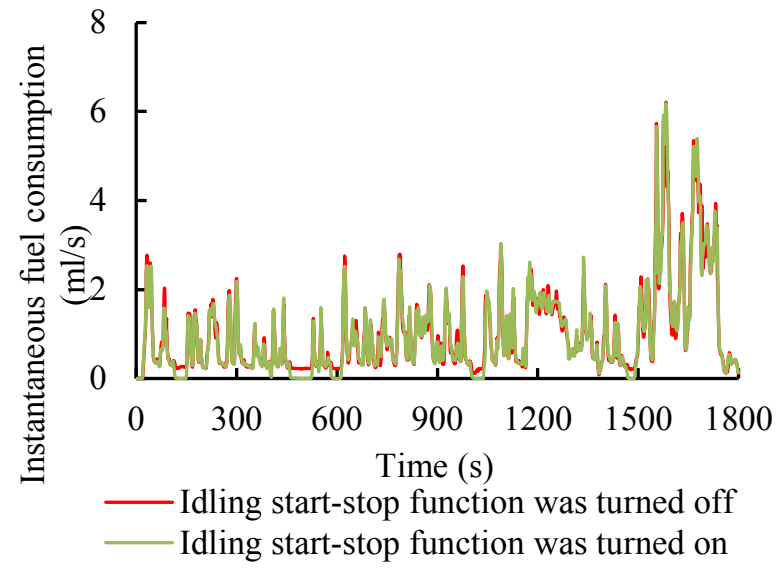

Fig. 5. Instantaneous fuel consumption of WLTC condition test. 


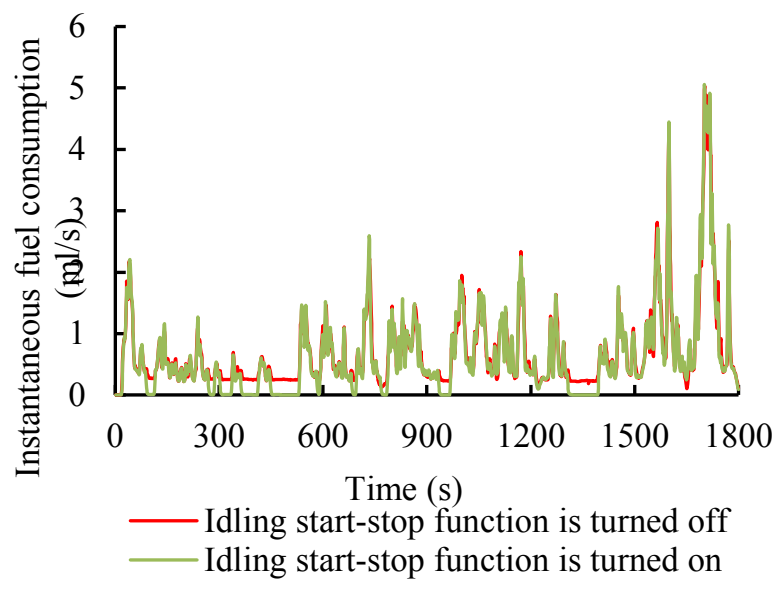

Fig. 6. Instantaneous fuel consumption of CLTC condition test.

When the idling start-stop function was turned on, after the idling segment ended, more fuel will be injected into the engine to ensure that the engine started again smoothly.This will cause the mixture to become richer and the excess air coefficient to decrease. Figure 7 shows the instantaneous excess air coefficient of the 4th idling segment in NEDC condition test of vehicle 2 . At the moment of vehicle starting, the excess air coefficient value reached 0.79 when the idling start-stop function was turned on and 1.04 when the idling start-stop function was turned off. The amount of fuel saved during idling and the amount of fuel injected in the restart phase after the end of idling segment determine the amount of fuel saved in a single idling segment. The amount of fuel saved during idling is determined by the average fuel consumption per second when idling and the duration of a single idling segment . In conclusion, it can be seen that the average fuel consumption per second when idling, the duration of single idling and the amount of fuel injected in the restart phase after the end of idling segment are the three determinants of the fuel saving benefit of single idling start-stop function [9].

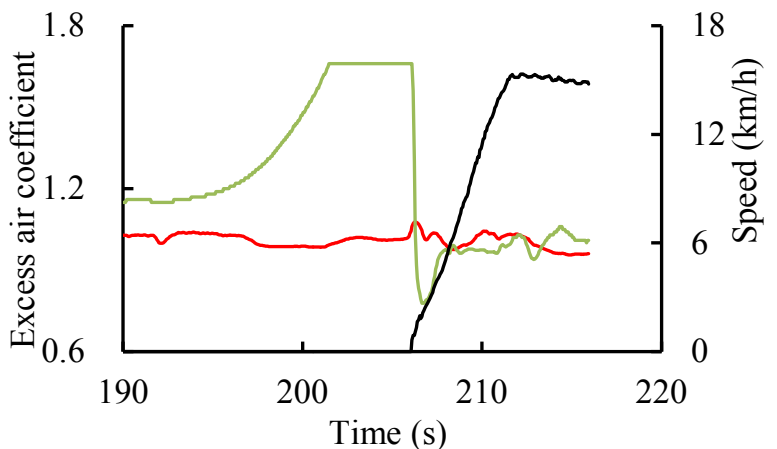

- Idling start-stop function was turned off Idling start-stop function was turned on Vehicle speed

Fig. 7. Instantaneous excess air coefficient.

Table 4 shows the fuel consumption results of different cycles when the idling start-stop function was turned on and off, and figure 8 shows the fuel saving rate of the idling start-stop functions in different cycles. It can be seen from the above that the trend of fuel saving rate of the three test vehicles was the same, and the fuel saving rate of the three 
cycles from low to high was WLTC condition, NEDC condition and CLTC condition. And the fuel saving rate of CLTC condition was $1.55 \%$ and $3.06 \%$ higher than that of NEDC condition and WLTC condition on average.

Because the idling times and idling proportion of the WLTC condition are the lowest among the three kinds of cycles, and the duration of the 3rd, 5th and 9th idling segments in the WLTC condition are very short, respectively $6 \mathrm{~s}, 3 \mathrm{~s}$ and $6 \mathrm{~s}$. As a result, the engine was restarted immediately after stopping. The amount of fuel injected during engine restart phase may offset the amount of fuel saved during shutdown, resulting in no fuel saving benefit for these three idling segments. Such idling segments reduce the comprehensive fuel saving rate of WLTC condition. To sum up, the low idling proportion and short idling segments result in low fuel saving rate of WLTC condition .The idling proportion of NEDC condition and CLTC condition is all about $23 \%$, but the fuel saving rate of CLTC condition is $1.55 \%$ higher than that of NEDC condition. This is because the idling start-stop function of the first idling segment of the NEDC condition did not work because the engine coolant temperature was too low, which reduces the fuel saving rate of the whole NEDC condition. Moreover, the idling times of NEDC condition are more than CLTC condition, resulting in more engine restart times in NEDC condition than CLTC condition, so that the total fuel injection amount in the restart phase is higher than CLTC condition. When the average fuel consumption per second is the same at idling, the longer the idling duration is, the more fuel is saved. The proportion of idling segments over $45 \mathrm{~s}$ in CLTC condition is $54.65 \%$, much higher than $28.51 \%$ in NEDC condition. The above reasons together lead to the lower fuel saving rate of NEDC condition than CLTC condition. In summary, it can be seen that the cycle which has higher idling proportion, fewer idling times, and more idling segments with a longer duration, the higher the fuel saving rate of the idling start-stop function.

Table 4. Fuel consumption results of different cycles.

\begin{tabular}{ccccccc}
\hline & \multicolumn{2}{c}{ NEDC(L/100KM) } & \multicolumn{2}{c}{ WLTC(L/100KM) } & \multicolumn{2}{c}{ CLTC(L/100KM) } \\
& $\begin{array}{c}\text { Function } \\
\text { on }\end{array}$ & $\begin{array}{l}\text { Function } \\
\text { off }\end{array}$ & $\begin{array}{l}\text { Function } \\
\text { on }\end{array}$ & $\begin{array}{l}\text { Function } \\
\text { off }\end{array}$ & $\begin{array}{l}\text { Function } \\
\text { on }\end{array}$ & $\begin{array}{l}\text { Function } \\
\text { off }\end{array}$ \\
\hline 1 & 6.58 & 6.80 & 6.41 & 6.54 & 6.91 & 7.26 \\
2 & 7.72 & 8.02 & 7.49 & 7.67 & 8.10 & 8.55 \\
3 & 9.14 & 9.81 & 8.93 & 9.39 & 9.99 & 10.90 \\
\hline
\end{tabular}

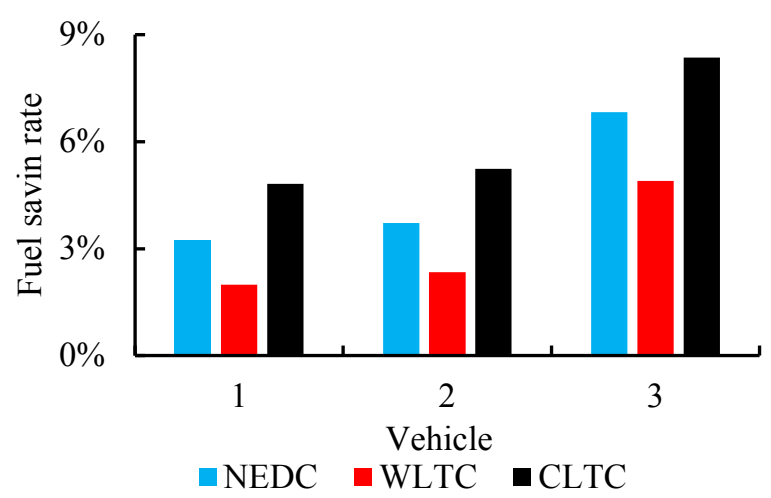

Fig. 8. Fuel saving rate of the idling start-stop function. 
In addition to the impact on the fuel consumption of the vehicles, the idling start-stop function also affects vehicles emissions. Figures 9 to 11 show the gaseous pollutant emissions when the idling start-stop function was turned on and off under different cycles. The idling start-stop function has little effect on the emission of gaseous pollutants. The maximum deviations of THC, CO, and NOx emissions are $3.15 \mathrm{mg}, 28.62 \mathrm{mg}$, and $1.98 \mathrm{mg}$, respectively. The gaseous pollutant emissions of all test vehicles meet the China 6 emission standard limits. Except for individual tests, the THC and CO emissions of the three test vehicles in different cycles when the idling start-stop function was turned on are higher than the emissions when the idling start-stop function was turned off, while NOx emissions are lower than those when the idling start-stop function was turned off.

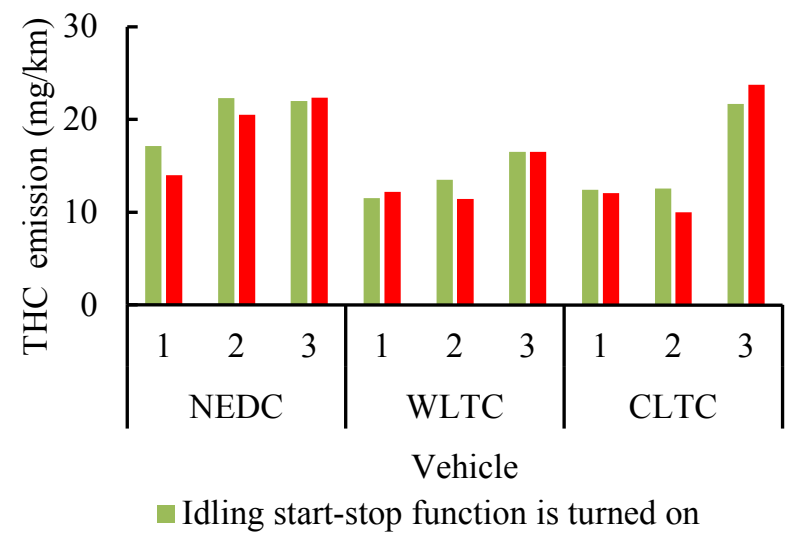

Fig. 9. THC emission.

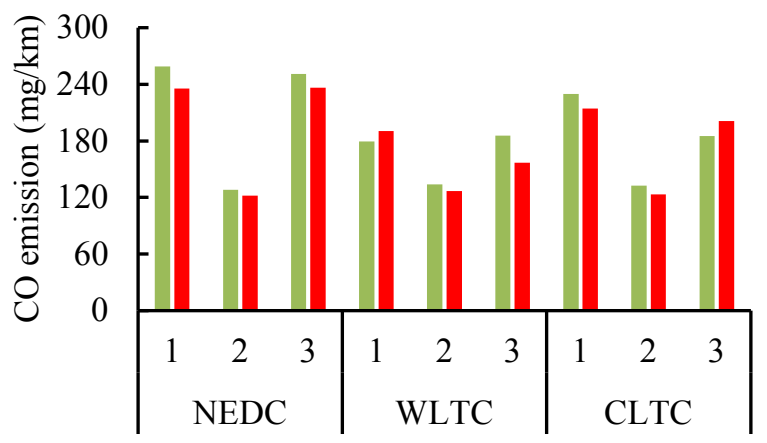

Vehicle

Idling start-stop function is turned on

- Idling start-stop function is turned off

Fig. 10. CO emission. 


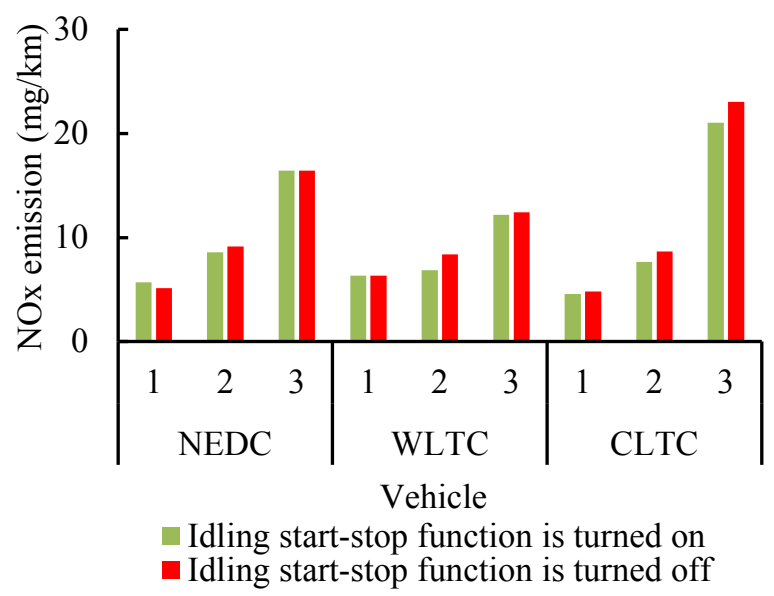

Fig. 11. NOx emission.

When the idling start-stop function worked normally, the engine was shut down at idling, after the idling segment ends, more fuel will be injected into the engine during the vehicle restart phase to ensure that the engine restarted smoothly. This caused the mixture to become richer, and at this time, it promoted pollutant emissions increase [10]. It can be seen that the impact of the idling start-stop function on vehicle pollutant emissions is mainly determined by the amount of pollutant emission reduction at idling and the amount of pollutant increase at the engine restart phase after idling. Taking the 4th idling segment of NEDC condition test of vehicle 2 as an example, the influence of idling start-stop function on vehicle pollutant emission was analyzed.Table 5 shows the amount of pollutant emission reduction during this idling segment and the amount of pollutant increase during the engine restart phase. It can be seen from the table that the reduction amounts of THC and $\mathrm{CO}$ in the idling phase are smaller than the increase in the restart phase, but the NOx reduction is larger than the increase in the restart phase. As a result, the idling start-stop function reduced NOx emission, but increased THC and $\mathrm{CO}$ emissions. The current engine calibration technology is quite mature, even if the engine does not stop during the idling phase, there are very few emissions, so the emission reduction during the idling phase is relatively small. However, when the engine is restarted later, the air-fuel mixture in the cylinder is enriched, the excess air coefficient in the local space deviates from 1, the cylinder wall temperature is insufficient and other factors promote the generation of pollutants, and instantaneous spikes in pollutant emissions occur, resulting in the increase amounts of THC and $\mathrm{CO}$ in the restart phase are greater than the reduction amounts in the idling phase. However, the state of hypothermia and hypoxia relatively inhibited the generation of NOx, resulting in a relatively small increase in NOx throughout the restarting phase. Eventually, the increase was less than the emission reduction during the idling phase.

Table 5. Emission reduction and increase caused by idling start-stop function.

\begin{tabular}{cccc}
\hline & $\begin{array}{c}\text { Reduction amount } \\
(\mathrm{mg})\end{array}$ & $\begin{array}{c}\text { Increase amount } \\
(\mathrm{mg})\end{array}$ & $\begin{array}{c}\text { Difference }(\mathrm{m} \\
\mathrm{g})\end{array}$ \\
\hline CO & 0.1187 & 0.86081 & -0.7421 \\
THC & 0.0824 & 0.619792 & -0.5374 \\
NOx & 0.2741 & 0.16062 & 0.1135 \\
\hline
\end{tabular}




\section{Conclusion}

(1) The idling times and total idling time of WLTC condition are the lowest among the three cycles. The idling times and total idling time of NEDC condition are almost the same as CLTC cycle, but the single idling segment duration of NEDC condition is mostly 20s to $25 \mathrm{~s}$, while the single idling segment which duration exceeding $45 \mathrm{~s}$ accounted for more than $50 \%$ of the total idling time of CLTC condition.

(2) The average fuel consumption per second during idling, the duration of single idling segment and the amount of fuel injected in the restart phase after the end of idling are the three determinants of the fuel saving benefit of single idling start-stop function. The fuel saving rate of idling start-stop function from low to high is WLTC condition, NEDC condition and CLTC condition. The fuel saving rate of CLTC condition is $1.55 \%$ and $3.06 \%$ higher than that of NEDC condition and WLTC condition on average.

(3) In all test cycles, the idling start-stop function has little effect on vehicle gaseous pollutant emissions. The maximum deviations of THC, CO, and NOx emissions are $3.15 \mathrm{mg}$, $28.62 \mathrm{mg}$, and $1.98 \mathrm{mg}$, respectively. The gaseous pollutant emissions of all test vehicles meet the China 6 emission standard limits. Except for individual tests, the THC and CO emissions of the three test vehicles in different cycles when the idling start-stop function was turned on are higher than the emissions when the idling start-stop function was turned off, while NOx emissions is lower than those when the idling start-stop function was turned off.

\section{Reference}

1. Li Jiahui, Li Daohua. On the current situation of the automobile exhaust pollution in china and the eeasures for its prevention and control [J]. Journal of Neijiang Normal University, 2009, 24 (8): 70-752.

2. Cai Jian, Feng Chunyan. Exploration on the harm and the countermeasures of automobile exhaust pollution [J]. Journal of Guangdong University of Petrochemical Technology, 2008, 18 (3): 40-43.

3. Zheng Jingzu. The harm of automobile exhaust [J]. Natural Science, 2004 (8): 46-49.

4. Liu Weiyi. Countdown to the introduction of Chinese working conditions [J]. Beijing Business Today, 2019-11-12.

5. Chen Xiujuan. The difference between the fuel consumption in the working condition and the actual fuel consumption is 30\% [J]. Automotive Observer, 2019 (3): 92-94.

6. Liu Zhiyong, Liu Xuezhu, He Yuguo. Research and Practice on Energy Saving Effects Evaluation Methods ofIdle Start-Stop [J]. China Automotive, 2018 (8): 37-42.

7. Cai Yingui, Bei Shaoyi, Wang Wei. Performance co-simulation analysis of engine idle start-stop system [J] Modern Manufacturing Engineering, 2017 (1): 19-23.

8. Li Zhenlei, Lin Yi, Gong Xu. An experimental study on the mild hybrid electric car with smart start-stop system[J]. Automotive Engineering, 2010, 32 (8): 654-658.

9. Moritaka Matsuura, Koji Korematsu, Junya Tanaka. Fuel consumption improvement of vehicles by idling stop [J]: SAE Paper2004-01-1896, 2004.

10. Michael Duoba, Henry Ng, Robert Larsen. In-Situ Mapping and Analysis of the Toyota Prius HEV Engine [J] SAE Technical Paper 2000-01-3096, 2000. 